\title{
Influencia del Compromiso y las Normas Sociales en la Intención del Cumplimiento Tributario
}

\author{
Nancy J. Barberan(1), Miguel A. Bustamante ${ }^{(2) \star}$ y Roberto M. Campos ${ }^{(2)}$ \\ (1) CENTRUM Católica Graduate Business School, Lima, Perú (e-mail: njbarberan@pucp.pe) \\ (2) Facultad de Economía y Negocios, Universidad de Talca, Av. Lircay s/n, Talca-Chile. (e-mail: mabu@utalca.cl) \\ * Autor a quien debe ser dirigida la correspondencia.
}

Recibido Sep. 25, 2018; Aceptado Dic. 5, 2018; Versión final Dic. 20, 2018, Publicado Jun. 2019

\begin{abstract}
Resumen
Se determina la relación entre el compromiso y las normas sociales con la intención del cumplimiento tributario. La investigación se realizó con un enfoque cuantitativo, de diseño no experimental y alcance correlacional. Se identificaron como variables independientes el compromiso y norma social y como dependiente la intención del cumplimiento tributario. Se trabajó con una muestra de microempresas determinada aleatoriamente, localizadas en la ciudad de Guayaquil, Ecuador. Del análisis realizado se pudo determinar que la relación entre el compromiso y la intención del cumplimiento tributario es positiva y significativa, mientras que la relación entre norma social e intención de cumplimiento tributario es positiva pero no-significativa. Se pudo demostrar además, que el sector económico modera la relación entre el compromiso y la intención del cumplimiento tributario, existiendo una relación más fuerte entre estas variables para el sector servicios. Se concluye que el compromiso influye en la intención del cumplimiento tributario de las microempresas, no encontrándose evidencia suficiente de que la norma social tenga influencia significativa.
\end{abstract}

Palabras clave: compromiso; norma social; intención; cumplimiento tributario

\section{Influence of Commitment and Social Norms in the Intention of Tax Compliance}

\begin{abstract}
The relationship between the commitment and social norms in the tax compliance intention has been determined. The research has a quantitative approach, with a non-experimental design and a correlational scope. The independent variables are the commitment and social norm and the dependent variable is the tax compliance intention. The sample was built based in a random sample of the micro-business that resides in the city of Guayaquil, Ecuador. It was determined that the relationship between the commitment and the intention of tax compliance is positive and significant, while the relationship between social norm and tax compliance tax compliance intention is positive but no-significant. It was shown that the economic sector moderates the relationship between commitment and the intention of tax compliance, existing a stronger relationship between these variables in the services sector. Based on the results of this study, it is concluded that the commitment influences the intention of the tax compliance of the micro-business, not finding enough evidence that the social norm has any influence on the dependent variable.
\end{abstract}




\section{INTRODUCCIÓN}

Las situaciones tributarias han sido estudiadas desde los orígenes de la teoría económica (Gangl et al., 2015), y se analiza el cumplimiento tributario verificando cómo se comportan los individuos (Langham et al, 2012). Así también se ha estudiado, la relación entre la obligación tributaria y el comportamiento de los empresarios. Según Allingham y Sandmo (1972), la conducta tributaria es influida por los miembros de la familia y, en algunos casos, estimulando la conducta evasora, de manera que el contribuyente pagará sus impuestos cuando existe mayor riesgo de detección y, por el contrario, elegirá evadir, si percibe que no hay posibilidades de ser detectado. Los individuos tienen actitudes positivas cuando se sienten identificados con los tributos (Oberholzer y Stack, 2014), o bien son negativas si tienden a la corrupción y a la evasión. Desde una perspectiva extrema, a la corrupción se la define como el mal uso del poder público para obtener algún beneficio privado o político (Laso, 2010). Se confrontan de esta manera, las conductas de cumplimiento tributario con las conductas de evasión. Sin embargo, éstas dependen del tamaño y ubicación de la empresa Gobena y Van Dijke (2016), produciéndose con mayor intensidad en las pequeñas y medianas empresas, PYMES (DeBacker et al., 2015).

Por otra parte, la intención de cumplimiento tributario (Langham et al., 2012) es analizada por la teoría de la acción razonada (Fishbein y Ajzen, 1975; Ajzen y Fishbein, 1980), que la estudia a partir de las intenciones y de las actitudes respecto de una norma. La norma sin embargo, domina las decisiones de cumplimiento y enlaza las intenciones con el comportamiento real (Murphy et al., 2016). En consecuencia, las normas sociales influyen en la toma de decisiones individuales y permiten comprender en qué medida los contribuyentes evaden los impuestos o se coluden (Cohen et al., 2015). La conducta, puede ser activada de manera intrínseca, por el deseo de contribuir a un bien público superior, el cual ocurre cuando los individuos se sienten identificados con los tributos (Oberholzer y Stack, 2014). Por el contrario, los individuos pagan impuestos por motivación extrínseca, cuando el estímulo a la conducta proviene de su preocupación por las eventuales sanciones que les pudieran afectar por el hecho de no pagar (DeBacker et al., 2015). Complementariamente, si se analiza desde la perspectiva de la norma social, ésta incide sobre la intención (Kirchler, 2007; Brizi et al., 2015) y consecuentemente sobre la evasión tributaria; sin embargo, no se ha evidenciado que incremente o disminuya la evasión (Fellner et al., 2013).

En la posición opuesta, uno de los factores sociales que puede ayudar a explicar mejor el incumplimiento tributario (Williams y Krasniqi, 2017) es que no hay relación entre norma social y cumplimiento tributario (Wenzel, 2004). La norma social, por su parte, se define sobre la base de directrices compartidas de comportamiento apropiado y aceptado por un colectivo, determina reglas o estándares que son asumidos por los miembros del grupo a quienes guía o restringe pero, sin la fuerza de la ley (Cialdini y Trost 1998, p. 152; citado por Bobek et al., 2013), pudiendo derivar de intereses, valores y actitudes particulares propias del grupo social. Desde la perspectiva de la norma social, ésta regula desde la cultura el grado de rechazo o de aceptación de conductas (Kogler et al., 2015), en consecuencia, la aceptación de los individuos dentro de un grupo social afecta el cumplimiento tributario y es mayor cuando existe motivación intrínseca (Williams y Krasniqi, 2017) la cual se determina por parámetros de costumbre social y motivaciones colectivas que dan sentido a valores personales y normas sociales (De Backer et al., 2015) e inciden en las decisiones de cumplimiento de los contribuyentes (Bobek et al., 2013).

Entre los factores estudiados en el cumplimiento tributario, el compromiso es esencial y refleja creencias y sentimientos de obligación moral para cumplir obligaciones tributarias (Braithwaite, 2003). Para Bornman y Wessels (2017) existe una correlación positiva entre el compromiso y la intención del cumplimiento tributario (Gangl et al., 2015) puesto que influye en las decisiones de los individuos (Mittone y Saredi, 2016) y se caracteriza por la responsabilidad del individuo respecto del sistema tributario. Por su parte, la intención, se corresponde con la disposición de una persona para llevar a cabo un determinado comportamiento que se evidencia en el cumplimiento tributario (Ajzen y Fishbein, 1980). En síntesis, el cumplimiento tributario potencia el acatamiento de términos, requisitos y formalidades validados por la norma social (Kogler et al., 2015) e implica compromiso (Braithwaite, 2003) e intención de cumplimiento tributario (Fishbein y Ajzen, 1975; Ajzen, y Fishbein, 1980). En consecuencia, sobre la base de la teoría de la acción razonada, el presente trabajo busca determinar las relaciones que se presentan entre los factores normas sociales, compromiso e intención de cumplimiento tributario, a fin de verificar su comportamiento entre las empresas pequeñas localizadas en la ciudad de Guayaquil, Ecuador.

\section{METODOLOGÍA}

Los materiales y métodos utilizados en seis subsecciones: (i) Fuentes de información y variables en estudio, (ii) Hipótesis de trabajo, (iii) Análisis de los datos, (iv) Análisis factorial confirmatorio, (v) Validez convergente, y (vi) confiabilidad y procesamiento de datos 


\section{Fuentes de información y variables en estudio}

La población en estudio estuvo conformada por microempresas ubicadas en la ciudad de Guayaquil, registradas en la base de datos de Registro Único de Contribuyentes y perteneciente a los sectores inmobiliarias, comercio, servicios, construcción, agricultura, ganadería, silvicultura y pesca e industrias manufactureras. La muestra determinada a priori previó una varianza máxima de $50 \%$ considerando una distribución normal de la población. Se contempló un intervalo de confianza $\sigma \mathrm{P}$ de $95 \%$ (1-b probabilidad de error) equivalente a una longitud aproximada $Z=1,96$ y se consideró un error de estimación de $5 \%$ como el máximo dispuesto a admitir para el estudio, alcanzándose una muestra representativa estimada de 384 unidades. El estudio adopta la teoría de la acción razonada (Fishbein y Ajzen, 1975; Ajzen y Fishbein 1980). Se identificó como variables independientes compromiso y norma social (Kogler et al., 2015). Los instrumentos utilizados fueron el propuesto por Ajzen y Fishbein (1980) en referencia a la intención, el de Kogler et al. (2015) respecto de la norma social y el de Braithwaite (2003) referido al compromiso. Los sujetos fueron los administradores de las empresas y los personales asignados al área de impuestos. Como variable dependiente se definió la intención de cumplimiento tributario (Ajzen y Fishbein, 1980). Por otro lado se observa que Gangl et al. (2015) y Gobena y Van Dijke (2016), dejan abierta la posibilidad de realizar investigaciones tomando en consideración caracteristicas adicionales al compromiso como son el tamaño de las empresas.

\section{Hipótesis de trabajo}

Considerando que, desde la perspectiva teórica de la acción razonada (Fishbein y Ajzen, 1975; Ajzen y Fishbein, 1980), la variable compromiso resulta ser un inductor clave puesto que la intención de una conducta depende precisamente del compromiso, como por ejemplo, la de no cometer fraudes tributarios (Yusof y Lai, 2014), lo que sugiere para el presente estudio la siguiente primera hipótesis de trabajo:

H1: Existe una relación significativa entre el compromiso y la intención del cumplimiento tributario en las microempresas de Guayaquil, Ecuador. Por otra parte, dado que algunos estudios basados en la teoría de la acción razonada, determinaron que las normas afectan la intención del cumplimiento tributario (Ho et al., 2013; Kogler et al., 2015) y que además éstas dominan las decisiones de cumplimiento de los contribuyentes (Murphy et al., 2016), se deriva la siguiente segunda hipótesis: H2: Existe una relación significativa entre la norma social y la intención del cumplimiento tributario en las microempresas de Guayaquil, Ecuador. Por último, considerando la pertenencia social de las empresas a sus respectivos sectores industriales y que dentro del mismo se estructuran conforme al tamaño de las empresas y que estos factores son determinantes en el cumplimiento tributario (Lignier et al., 2014), se genera la tercera hipótesis del presente trabajo: $\mathrm{H3}$ : El sector económico modera la relación entre el compromiso y la norma social con la intención del cumplimiento tributario en las microempresas de Guayaquil, Ecuador.

\section{Análisis de los datos}

La investigación es de tipo cuantitativo, de diseño no experimental, de corte transversal y alcance correlacional. Se aplicó supuestos de normalidad y homogeneidad de la varianza para justificar el uso de técnicas de análisis paramétrico-multivariado (Pérez y Medrano, 2010). Además, se optó por el supuesto de linealidad, como implícito en las técnicas multivariantes basadas en medidas de correlación (Freiberg et al., 2013) el cual fue evaluado mediante el análisis de la existencia de algún efecto no lineal entre las variables a través de gráficos de dispersión entre las variables de estudio (Chion y Charles, 2016).

\section{Análisis factorial confirmatorio, AFC}

Se realizaron los pasos previos sugeridos por Kaplan, (Pérez y Medrano, 2010) antes de aplicar AFC y se tuvo en consideración algunos criterios de decisión clave (Chion y Charles, 2016) como considerar un mínimo de 3 ítems por factor si éstos no están correlacionados o un mínimo de 2 si los factores se correlacionan. Se utilizó la matriz de correlaciones producto-momento de Pearson (Lloret-Segura et al, 2014) y la matriz de varianza-covarianza para prever efectos de asimetría de las distribuciones de los ítems (Izquierdo et al., 2014). Se aplicó AFC mediante el método de Máxima Verosimilitud ML (Lloret-Segura et al, 2014) el cual permitió evaluar los índices de bondad de ajuste del modelo propuesto cuantificándolos de manera conservadora (Chion y Charles, 2016). Los análisis de bondad de ajuste se realizaron considerando el índice absoluto estadístico-radio de verosimilitud de chi-cuadrado (CMIN/DF $\leq 3$ ), (Chión y Charles, 2016) el índice de bondad de ajuste GFI ( $\geq 0,9)$, (Freiberg et al., 2013) el índice de error cuadrático medio de aproximación RMSEA ( $\leq$ $0,05)$ (Torres, 2011), además del índice de ajuste incremental AGFI $(\leq 0,8)$, el índice no normado de ajuste de Tucker Lewis TLI $(\leq 0,9)$ (Freiberg et al., 2013) y el índice normado de ajuste NFI $(\leq 0,9)$. (Chion y Charles, 2016). 


\section{Validez convergente, confiabilidad y procesamiento de datos}

La validez convergente se verificó evaluando si el coeficiente estimado de cada indicador sobre su constructo subyacente es significativo ( $p$-valor $<0,05$ ) y confirmado mediante el agrupamiento coherente de ítems en dimensiones representativas (Freiberg et al., 2013). Finalmente, para medir la fiabilidad del instrumento se aplicó el alfa de Cronbach y el índice de confiabilidad compuesto que propone Chion y Charles (2016), donde la validez discriminante es evaluada a través de una prueba t-student planteando como hipótesis nula que la correlación entre constructos es igual a uno. En el procesamiento de datos se utilizó el software estadístico SPSS V20, para los análisis descriptivos de la muestra y para el modelamiento mediante ecuaciones estructurales se utilizó AMOS de SPSS (Freiberg et al., 2013) aplicando el método de Máxima Verosimilitud, ML.

\section{RESULTADOS}

Se presenta el análisis descriptivo de la muestra y de los datos perdidos y atípicos, el análisis factorial confirmatorio y modelo estructural con su respectivo análisis de bondad de ajuste.

\section{Análisis descriptivo de la muestra}

La muestra final post hoc obtenida fue de $453 \geq 384$ determinada a priori lograndose un parámetro de no centralidad de probabilidades 3,229, un valor t critico de 1,648 que define una región crítica o de rechazo y una potencia de 0,943 que define la probabilidad de la prueba estadística para rechazar una hipótesis nula falsa. Además se redujo el error estimado ex ante de 5\% a 3,87\% post hoc. De los encuestados, el $63,4 \%$ corresponden al género femenino y $36.6 \%$ al masculino, cuya edad se encuentra concentrada en los rangos $31-40$ y $20-30$ años con el $42,4 \%$ y $36,2 \%$ respectivamente. El sector económico al que pertenecen con mayor representatividad corresponde los sectores de servicios, alcanzando un 31,8\%, inmobiliario con el $28,9 \%$ y comercio con el 19,9\%. La mayoría de encuestados (76\%), ocupan cargos no directivos frente a un $24 \%$ que laboran como Gerente o Jefes; de ellos, el $26,5 \%$ y el $24,7 \%$ han laborado en sus instituciones entre seis y 10 años y entre dos y seis años respectivamente, $20,8 \%$ ha laborado entre 10 y 20 años.

\section{Análisis de datos perdidos y valores atípicos}

Se realizó un análisis de la muestra mediante diagramas de caja y examen de residuales a fin de determinar anomalías (Freiberg et al., 2013). Los resultados se muestran en la Tabla 1. En general, no se encontraron desviaciones importantes que indiquen problemas de homocedasticidad en las preguntas correspondientes a la variable dependiente intención de cumplimiento tributario. Además, luego de analizar los gráficos de dispersión entre las variables independientes y dependientes, se verificó que no existen problemas de no linealidad que pudieran afectar los análisis previstos. En la fase de depuración de la muestra, se confirmó mediante el cálculo de la distancia de Mahalanobis $\left(D^{2}\right)$ (Freiberg et al., 2013), la no existencia de valores atípicos, datos perdidos y categorías con datos ausentes, se consideró un rango de entre $1 \%$ y $3 \%$, el cual se evaluó con el método de imputación por regresión. En la Tabla 1 se muestra que no existen valores de $D^{2}$ demasiado alejados del resto; además, en la columna $\mathrm{p}^{1}$, que representa la probabilidad de que una observación supere la distancia $\mathrm{D}^{2}$ asociada y donde se espera que tengan valores pequeños (Freiberg et al., 2013), no se observan valores que puedan considerarse atípicos. Finalmente, la prueba de Little mostró un valor de chi cuadrado $=226,485(p<0,05)$, que permite rechazar la hipótesis nula de que los datos responden a un proceso completamente aleatorio.

\section{Verificación de normalidad, confiabilidad y validez de las variables}

Se verificó el cumplimiento del criterio de normalidad multivariante cuyo coeficiente es de 59,43 $\leq 70$ sugerido por el coeficiente de Mardia (Freiberg et al., 2013) lo que permite asumir que el método ML produce los mejores resultados y se cumple con el criterio de normalidad multivariante. La escala de intención cumplimiento tributario es confiable, con un Alpha de Cronbach de 0,745 y se califica de aceptable (Freiberg et al., 2013). Las escalas de las variables compromiso y norma social, 0,635 y 0,613 respectivamente, se pueden considerar en grado de cuestionable pero aún confiables, puesto que muestran valores superiores a 0,5. Mediante el índice compuesto $\rho_{c}^{2}$ de Chion y Charles (2016), la confiabilidad de las variables muestra niveles aceptables. La variable compromiso con un $\rho_{c}{ }^{2}=0,80$, norma social con un $\rho_{c}{ }^{2}=0,60$ e intención de cumplimiento tributario con un $\rho_{c}{ }^{2}=0,96$. En consecuencia, las escalas en análisis cumplen con el criterio de validez convergente y la confiablidad de la variable dependiente es aceptable. 
Tabla 1: Resumen de valores perdidos y atípicos

\begin{tabular}{|c|c|c|c|c|c|c|c|}
\hline & \multirow[b]{2}{*}{$\mathrm{N}$} & \multirow[b]{2}{*}{ Media } & \multirow{2}{*}{\multicolumn{2}{|c|}{$\begin{array}{l}\text { Desviación } \\
\text { estándar }\end{array}$}} & \multicolumn{2}{|c|}{ Perdidos } \\
\hline & & & & & & Recuento & Porcentaje \\
\hline \multicolumn{2}{|c|}{ Compromiso_1 } & 441 & 4,94 & \multicolumn{2}{|c|}{0,249} & 12 & 2,6 \\
\hline \multicolumn{2}{|c|}{ Compromiso_2 } & 453 & 4,79 & \multicolumn{2}{|c|}{0,465} & 0 & 0,0 \\
\hline \multicolumn{2}{|c|}{ Compromiso_3 } & 452 & 4,49 & \multicolumn{2}{|c|}{0,830} & 1 & 0,2 \\
\hline \multicolumn{2}{|c|}{ Compromiso_4 } & 452 & 4,33 & \multicolumn{2}{|c|}{0,726} & 1 & 0,2 \\
\hline \multicolumn{2}{|c|}{ Compromiso_5 } & 452 & 4,25 & \multicolumn{2}{|c|}{0,720} & 1 & 0,2 \\
\hline \multicolumn{2}{|c|}{ Compromiso_6 } & 452 & 4,66 & \multicolumn{2}{|c|}{0,665} & 1 & 0,2 \\
\hline \multicolumn{2}{|c|}{ Compromiso_7 } & 448 & 2,26 & \multicolumn{2}{|c|}{0,940} & 5 & 1,1 \\
\hline \multicolumn{2}{|c|}{ Compromiso_8 } & 450 & 4,75 & \multicolumn{2}{|c|}{0,509} & 3 & 0,7 \\
\hline \multicolumn{2}{|c|}{ Norma_Social_1 } & 452 & 4,89 & \multicolumn{2}{|c|}{0,366} & 1 & 0,2 \\
\hline \multicolumn{2}{|c|}{ Norma_Social_2 } & 453 & 2,25 & \multicolumn{2}{|c|}{1,387} & 0 & 0,0 \\
\hline \multicolumn{2}{|c|}{ Norma_Social_3 } & 453 & 2,48 & \multicolumn{2}{|c|}{0,942} & 0 & 0,0 \\
\hline \multicolumn{2}{|c|}{ Norma_Social_4 } & 453 & 2,04 & \multicolumn{2}{|c|}{1,071} & 0 & 0,0 \\
\hline Norm & cial_5 & 452 & 4,39 & & & 1 & 0,2 \\
\hline Norm & cial_6 & 449 & 2,31 & & & 4 & 0,9 \\
\hline Inten & pl_Trib_1 & 453 & 4,92 & & & 0 & 0,0 \\
\hline Inten & pl_Trib_2 & 453 & 4,89 & & & 0 & 0,0 \\
\hline Inten & ipl_Trib_3 & 453 & 4,90 & & & 0 & 0,0 \\
\hline & & & Valore & típicos & & & \\
\hline $\mathrm{N}^{\circ}$ & $\mathrm{D}^{2}$ & $\mathrm{p}^{1}$ & $p^{2}$ & $\mathrm{~N}^{\circ}$ & $\mathrm{D}^{2}$ & $p^{1}$ & $\mathrm{p}^{2}$ \\
\hline 6 & 155,479 & 0,000 & 0,000 & 421 & 51,258 & 0,000 & 0,000 \\
\hline 433 & 97,527 & 0,000 & 0,000 & 376 & 50,943 & 0,000 & 0,000 \\
\hline 351 & 72,180 & 0,000 & 0,000 & 307 & 50,004 & 0,000 & 0,000 \\
\hline 168 & 62,227 & 0,000 & 0,000 & 55 & 49,707 & 0,000 & 0,000 \\
\hline 406 & 61,751 & 0,000 & 0,000 & 97 & 49,671 & 0,000 & 0,000 \\
\hline 320 & 61,546 & 0,000 & 0,000 & 177 & 47,226 & 0,000 & 0,000 \\
\hline 414 & 58,235 & 0,000 & 0,000 & 48 & 46,063 & 0,000 & 0,000 \\
\hline 176 & 57,893 & 0,000 & 0,000 & 33 & 43,449 & 0,000 & 0,000 \\
\hline 91 & 54,988 & 0,000 & 0,000 & 199 & 43,436 & 0,000 & 0,000 \\
\hline 104 & 52,664 & 0,000 & 0,000 & 76 & 41,501 & 0,001 & 0,000 \\
\hline 27 & 51,851 & 0,000 & 0,000 & 16 & 41,104 & 0,001 & 0,000 \\
\hline & & & & 140 & 40,456 & 0,001 & 0,000 \\
\hline
\end{tabular}

\section{Modelo estructural}

La tabla 2 detalla los pesos de regresión $\left(B_{1}\right)$ y los índices de regresión estandarizados $\left(B_{1 z}\right)$ de las variables latentes compromiso, norma social e intención de cumplimiento tributario.

Tabla 2: Modelo de medida del análisis estructural

\begin{tabular}{|c|c|c|c|c|}
\hline Ítems & $B_{1}$ & $B_{1 z}$ & $B_{2}$ & $p$ \\
\hline \multicolumn{5}{|c|}{ Modelo de Medida } \\
\hline Intención_Cumplimiento <--- Norma Social & 0,00 & 0,00 & 0,016 & 0,962 \\
\hline Intención_Cumplimiento <--- Compromiso & 0,21 & 0,18 & 0,076 & ** \\
\hline Norma_Social <--- Compromiso & $-0,01$ & $-0,11$ & 0,010 & * \\
\hline \multicolumn{5}{|c|}{ Variables } \\
\hline Norma_Social_6_R <--- Norma_Social & 1,00 & 0,54 & & \\
\hline Norma_Social_5 <--- Norma_Social & 0,17 & 0,14 & 0,063 & ** \\
\hline Norma_Social_4_R <--- Norma_Social & 0,71 & 0,55 & 0,087 & *** \\
\hline Norma_Social_3_R <--- Norma_Social & 0,67 & 0,58 & 0,079 & *** \\
\hline Norma_Social_2_R <--- Norma_Social & 1,36 & 0,81 & 0,153 & $* \star *$ \\
\hline Norma_Social_1 <--- Norma_Social & 0,01 & 0,03 & 0,024 & 0,572 \\
\hline
\end{tabular}


Tabla 2 (continuación)

\begin{tabular}{lcccc}
\hline \multicolumn{1}{c}{ Ítems } & $B_{1}$ & $B_{1 z}$ & $B_{2}$ & $p$ \\
\hline Intención_Cumplimiento_1 <--- Intención_Cumplimiento & 1,00 & 0,57 & & \\
Intención_Cumplimiento_2 <--- Intención_Cumplimiento & 1,09 & 0,68 & 0,104 & $* * *$ \\
Intención_Cumplimiento_3 <--- Intención_Cumplimiento & 1,47 & 0,88 & 0,155 & $* * *$ \\
Compromiso_8<--- Compromiso & 1,00 & 0,37 & & \\
Compromiso_7_R <--- Compromiso & 0,13 & 0,03 & 0,265 & 0,627 \\
Compromiso_6 <--- Compromiso & 1,23 & 0,35 & 0,187 & $* * *$ \\
Compromiso_5_R <--- Compromiso & 2,93 & 0,76 & 0,439 & $* * *$ \\
Compromiso_4<--- Compromiso & 2,93 & 0,76 & 0,440 & $* * *$ \\
Compromiso_3<--- Compromiso & 1,75 & 0,40 & 0,328 & $* * *$ \\
Compromiso_2<--- Compromiso & 0,92 & 0,37 & 0,179 & $* * *$ \\
Compromiso_1<--- Compromiso & 0,38 & 0,29 & 0,088 & $* * *$ \\
\hline \multicolumn{1}{c}{ Correlaciones entre constructos } & & & & $t$ \\
\hline Compromiso Tributario - Norma Social & & & & 78,18 \\
Compromiso Tributario - Intención Cumplimiento & & & & 331 \\
Norma Social - Intención Cumplimiento Tributario & & & & 91,18 \\
\hline
\end{tabular}

En general se determinaron índices $\beta_{1}$ y $\beta_{2}$ ubicados en sus respectivos rangos de normalidad. Los índices $\beta_{1 z}$ estandarizados se encuentran en el rango pertinente $(0-1)$ y los índices de varianza del error $\beta_{2}$ alcanzando valores bajos. Se observa que la mayoría de las relaciones son significativas ( $p$-valor) a diferentes índices, resultando no significativas las relaciones intención de cumplimiento tributario respecto de norma social $(0,962)$; norma social consigo misma $(0,572)$ y compromiso consigo mismo $(0,627)$. El modelo de medida considera la hipótesis nula de que la correlación entre constructos es igual a uno, además, se obtuvo, en todos los casos, que la prueba t-student supera el valor de 1,65. La correlación entre compromiso y norma social es de 78,18, entre compromiso e intención de cumplimiento tributario es de 331 y entre norma social e intención de cumplimiento tributario alcanza a 91,18, permitiendo rechazar la hipótesis nula y verificar que se cumple la validez discriminante. Se observa que la relación entre compromiso e intención de cumplimiento tributario es positiva y significativa $(0,21, p$-valor $<0,05)$; sin embargo, la relación Norma Social respecto de Compromiso es inversa $(-0,01)$ y significativa al $10 \%$. Por otro lado, la relación entre las variables norma social e intención de cumplimiento tributario es positiva pero no significativa $(0,00, p$-valor $>0,05)$.

\section{Bondad de ajuste}

Los resultados en la Tabla 3 muestran que, en la variable compromiso tributario, si bien el valor Chi-cuadrado no está dentro del umbral requerido ( $p$-valor > 0,002), los índices GFI y AGFI están dentro de los rangos especificados $(0,962$ y 0,925$)$. Por su parte, el valor de RMSEA $(0,081)$ se ubica en la parte alta de su rango, además del índice CMIN/DF que muestra un valor en el rango requerido $(3,982)$; no obstante, es importante acotar que, no existe un consenso claro sobre los valores límite para estos índices. Para la variable norma social, los índices GFI y AGFI están dentro de los umbrales requeridos (0,90 y 0,945), con un RMSA adecuado $(0,078)$ el que a su vez se ratifica con el índice CMIN/DF en el rango de aceptación requerido $(3,73)$, en consecuencia, el modelo presenta un buen ajuste para esta variable. Seguidamente, la variable intención de cumplimiento tributario presenta índices de bondad de ajuste GFI y AGFI adecuados alcanzando parámetros que se ubican en los rangos requeridos $(0,90$ y 0,80$)$.

Tabla 3: Índices de bondad de ajuste

\begin{tabular}{|c|c|c|c|c|c|c|c|c|}
\hline \multirow[b]{3}{*}{ Índice } & \multicolumn{6}{|c|}{ Validez Convergente } & \multicolumn{2}{|c|}{ Validez Discriminante } \\
\hline & \multicolumn{2}{|c|}{ Compromiso } & \multicolumn{2}{|c|}{ Norma Social } & \multicolumn{2}{|c|}{$\begin{array}{c}\text { Intención } \\
\text { Cumplimiento } \\
\text { Tributario }\end{array}$} & \multirow[b]{2}{*}{ Valor } & \multirow[b]{2}{*}{$\begin{array}{l}\text { Nivel de } \\
\text { Aceptación }\end{array}$} \\
\hline & Valor & $\begin{array}{l}\text { Nivel de } \\
\text { Aceptación }\end{array}$ & Valor & $\begin{array}{l}\text { Nivel de } \\
\text { Aceptación }\end{array}$ & Valor & $\begin{array}{l}\text { Nivel de } \\
\text { Aceptación }\end{array}$ & & \\
\hline Chi-cuadrado & 71,680 & $P<0,00$ & 33,61 & $P<0,002$ & 0 & & 442,506 & $p<0,002$ \\
\hline $\mathrm{Gl}$ & 18 & & 9 & & 0 & & 112 & \\
\hline GFI & 0,962 & $>0,90$ & 0,976 & $>0,90$ & 1 & $>0,90$ & 0,9 & $>0,90$ \\
\hline AGFI & 0,925 & $>0,80$ & 0,945 & $>0,80$ & - & $>0,80$ & 0,860 & $>0,80$ \\
\hline RMSEA & 0,081 & $<0,06$ & 0,078 & $<0,06$ & 0,05 & $<0,06$ & 0,081 & $<0,06$ \\
\hline CMIN/DF & 3,982 & $<5$ & 3,73 & $<5$ & - & $<5$ & 3,951 & $<5$ \\
\hline
\end{tabular}


Los índices de bondad de ajuste del modelo no presentaron buen ajuste en una primera instancia, lo que hizo necesario correlacionar las varianzas de error de las preguntas 1, 2 y 3 de la variable compromiso, con lo que se pudo determinar un mejor ajuste alcanzando valores en Chi-cuadrado con un valor $p$ aceptable $(0,002)$ al $10 \%$. Los índices de bondad de ajuste GFI y AGFI dieron resultados adecuados $(0,90$ y 0,860$)$, entregando un índice de error admisible $(0,081)$ y un coeficiente CMIN/DF en su rango de aceptación $(3,951)$.

\section{Modelo estructural}

El modelo que se muestra en la figura 1, incluye los efectos directos entre las variables latentes dando forma al modelo estructural. El modelo completo muestra un total de 17 variables observables que arroja un número de datos de $\left(17^{*} 18\right) / 2=153$. El número de incógnitas es 41 dando un total de $153-41=112$ grados de libertad. Este modelo presentó un ajuste aceptable, ya que la mayoría de los índices estuvieron dentro de los valores esperados

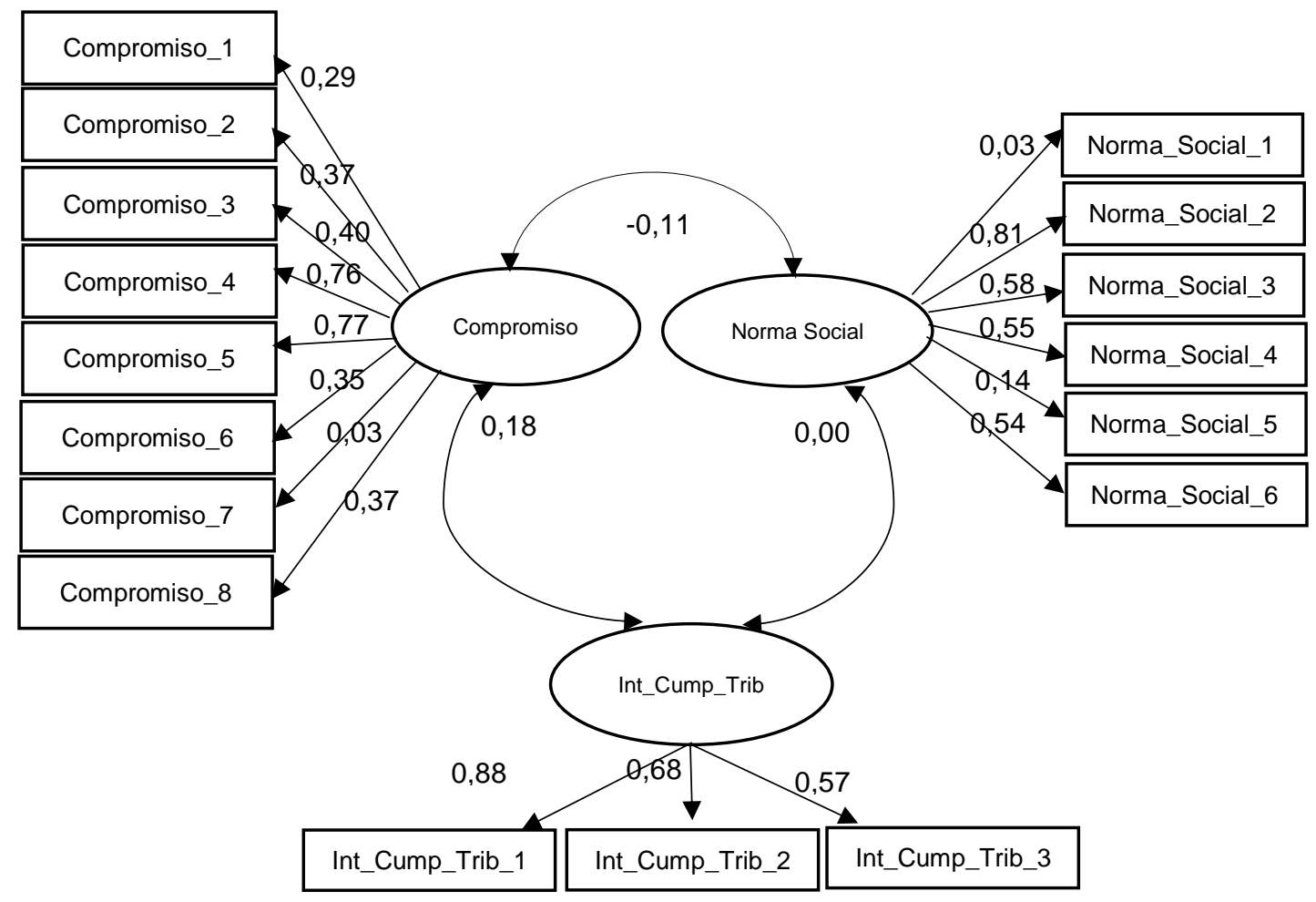

Fig. 1: Modelo estructural de intención de cumplimiento tributario

\section{Análisis con una variable moderadora}

Para ampliar el análisis, se incluyó la variable sector económico con el objetivo de determinar si ésta modera las relaciones entre las variables latentes (Tabla 4).

Tabla 4: Índices variable moderadora

\begin{tabular}{lllllll}
\hline & \multicolumn{2}{c}{ Sector Inmobiliario } & \multicolumn{2}{l}{ Sector Comercio } & \multicolumn{2}{c}{ Sector Servicios } \\
\cline { 2 - 7 } & Estimado & $p$-valor & Estimado & $p$-valor & Estimado & $p$-valor \\
\hline Compromiso -> Intención de Cumplimiento & 0,005 & 0,903 & $-0,086$ & 0,812 & 0,599 & 0,002 \\
Norma Social -> Intención de Cumplimiento & $-0,003$ & 0,874 & $-0,004$ & 0,879 & 0,069 & 0,055 \\
\hline
\end{tabular}

El análisis de la variable moderadora sector económico permitió determinar que los modelos son no invariantes o no equivalentes con una diferencia chi-cuadrado entre el modelo uno y el modelo dos de 19,359 con 10 grados de libertad, mayor al valor crítico de 18,31 a un nivel de significancia de 0,05 lo que demuestra que la diferencia chi-cuadrado es estadísticamente significativa. Además, el análisis de las relaciones entre las variables latentes de cada modelo permitió determinar que la relación entre el compromiso e intención de cumplimiento es más fuerte y significativa para el sector de servicios $(0,599 ; p$-valor $<0,05)$. En tanto que, en el sector inmobiliario la relación no fue significativa $(0,005 ; p$-valor $>0,05)$ y en el sector comercio fue negativa, aunque no significativa $(-0,086 ; p$-valor $>0,05)$. Finalmente, la relación entre norma social e intención de cumplimiento no fue significativa en ninguno de los sectores económicos. 


\section{DISCUSIÓN}

En general los índices de ajuste se encuentran dentro del umbral requerido, sin embargo el índice GFI está por debajo, aunque cerca del límite solicitado, dado que valores GFI y CFI cercanos a 0,9 puede considerarse un ajuste aceptable (Freiberg et al., 2013). Por otro lado, un valor RMSEA, que va de 0,08 a 0,1, indica un ajuste medio; mientras que valores mayores a 0,10 , un ajuste pobre del modelo, en consecuencia el modelo presenta índices que pueden ser considerados como adecuados (Freiberg et al., 2013). No obstante los resultados obtenidos que se ubican cercanos a los rangos óptimos y, tomando en cuenta que no existe un consenso claro sobre los valores límites que deben tener dichos índices, el modelo propuesto en este trabajo entrega una opción viable de relación entre las variables compromiso, normas sociales e intención de cumplimiento, y se puede señalar que el modelo presenta un ajuste aceptable (Freiberg et al., 2013).

Los resultados de la aceptación de H1, son concordantes con los de Bornman y Wessels (2017) quienes confirmaron una correlación positiva entre el compromiso y la intención del cumplimiento tributario, con los de Gangl et al. (2015) que establecen el compromiso como un factor relevante en el comportamiento tributario que influye en las decisiones de los individuos (Mittone y Saredi, 2016) y refleja los sentimientos de obligación moral para cumplir con los tributos (Braithwaite, 2003). Los resultados de rechazo de $\mathrm{H} 2$ a su vez son similares a los expuestos por Wenzel (2004) para quien las normas sociales no tendrían influencia significativa en la intención de cumplimiento tributario, y no evidencia que aumente o disminuya la evasión tributaria (Fellner et al., 2013). Se observa que los resultados logrados contradicen lo demostrado por Brizi et al. (2015) donde la orientación de valor social tiene una relación positiva con la intención de cumplimiento tributario; se oponen a lo determinado por DeBacker et al. (2015) quienes indican que la norma social tiene alta influencia en el pago de los tributos y colisiona con los argumentos de Ho et al., (2013) según los cuales la norma social influye en el comportamiento tributario y afecta la intención del cumplimiento tributario (Kogler et al., 2015; Cohen et al., 2015).

En general, las dimensiones independientes analizadas, inciden en las decisiones de cumplimiento de los contribuyentes (Murphy et al., 2016); por Yusof y Lai (2014) y ratifican los hallazgos de la teoría de la acción razonada (Fishbein y Ajzen, 1975; Ajzen y Fishbein, 1980), puesto que la norma social no determinan en forma significativas $(p=0,055)$ la intención de cumplimiento y abre un espacio a cometer fraudes tributarios, a pesar de considerarse entre las mas importantes de la norma social (Kirchler, 2007). Finalmente, el sector económico modera la relación entre compromiso e intención de cumplimiento tributario, siendo concordante con lo expuesto por Lignier et al., (2014) y Gobena y Van Dijke (2016).

\section{CONCLUSIONES}

Los resultados del análisis permiten aceptar la hipótesis $\mathrm{H} 1$, es decir que sí existe una relación significativa entre el compromiso y la intención del cumplimiento tributario en las microempresas de Guayaquil, Ecuador. Se rechaza sin embargo la hipótesis $\mathrm{H} 2$, dado que no existe una relación significativa entre la norma social y la intención del cumplimiento tributario. Se acepta $\mathrm{H} 3$ por cuanto la variable sector económico modera la relación entre compromiso e intención de cumplimiento tributario, siendo la relación más alta la que se presenta en el sector servicios; no obstante, no existe moderación de esta última con la norma social e intención de cumplimiento tributario. En general, las normas sociales no inciden de manera significativa en la intención de cumplimiento tributario y no se obtuvo evidencia de que el sector industrial modere las conductas de intención de cumplimiento tributario.

\section{REFERENCIAS}

Ajzen, I. y M. Fishbein, Understanding attitudes and predicting social behavior, Englewood Cliffs, NJ, Prentice-Hall (1980)

Allingham, M. y A. Sandmo, Income tax evasion: a theoretical analysis, Journal of Public Economics, 1(3-4), 323-338 (1972)

Bobek, D.D., A. M. Hageman y C. F. Kelliher, Analyzing the Role of Social Norms in Tax Compliance Behavior, Journal of Business Ethics, 115(3), 451-468 (2013)

Bornman, M. y J. Wessels, The role and dimensions of taxpayer commitment in tax compliance behaviour. EJournal of Tax Research, 15(3), 506-531 (2017)

Braithwaite, V., Dancing with tax authorities: Motivational postures and noncompliant actions, Ashgate, Aldershot (2003)

Brizi, A., M. Giacomantonio, B.M. Schumpe y L. Mannetti, Intention to pay taxes or to avoid them: The impact of social value orientation, Journal of Economic Psychology, 50, 22-31 (2015)

Chión, S., V. Charles, Analítica para la Modelación Estructural, Perú, Pearson Perú, 239-322 (2016)

Cohen, J., G. B. Manzon y V. L. Zamora, Contextual and Individual Dimensions of Taxpayer Decision Making, Journal of Business Ethics, 126(4), 631-647 (2015) 
DeBacker, J., B.T. Heim y A. Tran, Importing corruption culture from overseas: Evidence from corporate tax evasion in the United States, Journal of Financial Economics, 117(1), 122-138 (2015)

Fellner, G., R. Sausgruber y C. Traxler, Testing Enforcement Strategies in the Field: Threat, Moral Appeal and Social Information: Testing Enforcement Strategies in the Field, Journal of the European Economic Association, 11(3), 634$660(2013)$

Fishbein, M. e I. Ajzen, Belief, attitude, intention, and behavior: An introduction to theory and research (Reading, MA), Addison-Wesley (1975)

Freiberg, H.A., J. B. Stover, G. de la Iglesia, M. Fernández, Polychoric And Tetrachoric Correlations In Exploratory And Confirmatory Factorial Studies, Prensa Médica Latinoamericana - ISSN 1688-4094 Ciencias Psicológicas, VII (2), 151 $164(2013)$

Gangl, K., E. Hofmann y E. Kirchler, Tax authorities' interaction with taxpayers: A conception of compliance in social dilemmas by power and trust, New Ideas in Psychology, 37, 13-23 (2001)

Gobena, L. B. y M. Van Dijke, Power, justice, and trust: A moderated mediation analysis of tax compliance among Ethiopian business owners, Journal of Economic Psychology, 52, 24-37 (2016)

Ho, D., D.C. Ho y A. Young, Study of the Impact of Culture on Tax Compliance in China, A. Int'I Tax J., 39, 33 (2013)

Izquierdo, I., J. Olea y F. J. Abad Exploratory factor analysis in validation studies: Uses and recommendations, Psicothema, 26(3), 395-400 (2014)

Kirchler, E., The economic psychology of tax behaviour, Cambridge University Press (2007)

Kogler, C., S. Muehlbacher y E. Kirchler, Testing the "slippery slope framework" among self-employed taxpayers, Economics of Governance, 16(2), 125-142 (2015)

Langham, J., N. Paulsen y C. E. Härtel, Improving tax compliance strategies: Can the theory of planned behaviour predict business compliance? EJournal of Tax Research, 10(2), 364 (2012)

Laso, E., La confianza como encrucijada: cultura, desarrollo y corrupción, Athenea Digital, Revista de Pensamiento e Investigación Social, (17), 97-117 (2010)

Lignier, P., C. Evans y B. Tran-Nam, Tangled up in tape: The continuing tax compliance plight of the small and medium enterprise business sector, Austl. Tax F., 29, 217 (2014)

Lloret-Segura, S., A. Ferreres-Traver, A. Hernández-Baeza e I. Tomás-Marco, El análisis factorial exploratorio de los ítems: una guía práctica, revisada y actualizada, Anales de psicologíavol., 30(3), 1151-1169 (2014)

Mittone, L. y V. Saredi, Commitment to tax compliance: Timing effect on willingness to evade, Journal of Economic Psychology, 53, 99-117 (2016)

Murphy, K., B. Bradford y J. Jackson, Motivating Compliance Behavior among Offenders: Procedural Justice or Deterrence? Criminal Justice and Behavior, 43(1), 102-118 (2016)

Oberholzer, R. y E. M. Stack, Perceptions of taxation: A comparative study of different population groups in South Africa, Public Relations Review, 40(2), 226-239 (2014)

Pérez, E. y L. Medrano, Análisis Factorial Exploratorio: Bases Conceptuales y Metodológicas, Revista Argentina de Ciencias del Comportamiento, 2(1), 58- 66 (2010)

Torres, G., Una aproximación al análisis de competitividad aplicando la técnica del modelo de ecuaciones estructurales, TESCoalt, 13(31), 1-9 (2011)

Wenzel, M., An analysis of norm processes in tax compliance, Journal of Economic Psychology, 25(2), 213-228 (2004)

Williams, C.C. y B. Krasniqi, Evaluating the individual- and country-level variations in tax morale: Evidence from 35 Eurasian countries, Journal of Economic Studies, 44(5), 816-832 (2017)

Yusof, N.A.M. y Lai, M. L., An integrative model in predicting corporate tax fraud, Journal of Financial Crime, 21(4), 424-432 (2014) 
